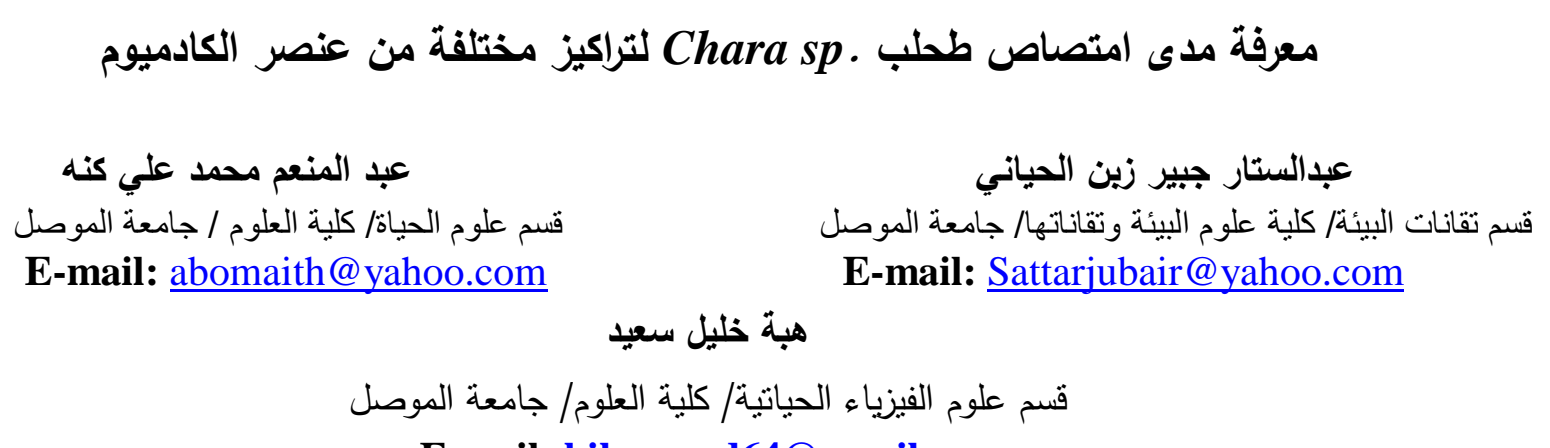

\title{
E-mail: hibasaeed64@gmail.com
}

(أستلم 2019/6/30 ؛ قُبل 2019/9/16)

\section{DOI: $\underline{\text { 10.33899/rjs.2020.164479 }}$}

\begin{abstract}
الملخص
تم استخدام الامتزاز الحيوي لأيون عنصر الكادميوم بواسطة الطحلب (Chara sp) حيث جفف وتم تشخيص المجاميع

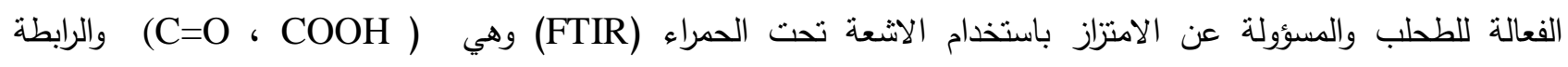
(C-H)

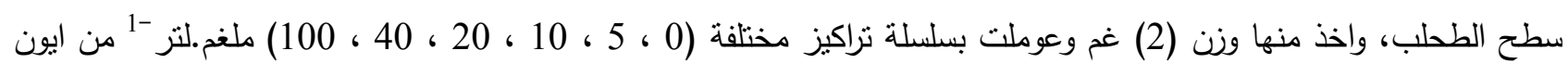
الكادميوم وبعدها حضنت المعاملات على درجة حرارة (298 ) كلفن، بعد انتهاء فترة التحضين تم استخلاص رواشح الاتزان وقدر ، فيها الكادميوم مع وصفها رياضيا حسب معادلات (لانكمير ذات السطح الواحد وذات السطحين، فرندلخ، دوبين، تمكن وبولاني) على التوالي للكادميوم، ووجد بان قيم معامل التحديد كانت مؤثرة لجميع المعادلات مما يعطي دلالة واضحة على على امكانية استخدام

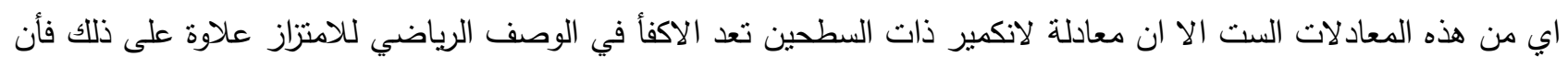
هذه المعادلة اظهرت نطابقاً كبيراً جدا لقيم الامتزاز الفعلية والقيم المحسوبة من هذه المعادلة وذللك لامتلاكها اقل خطأ قياسي

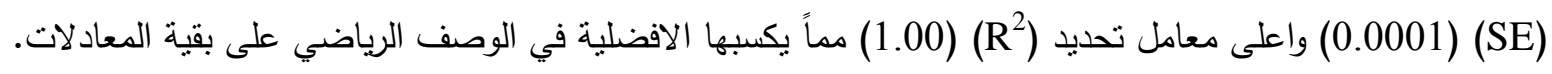

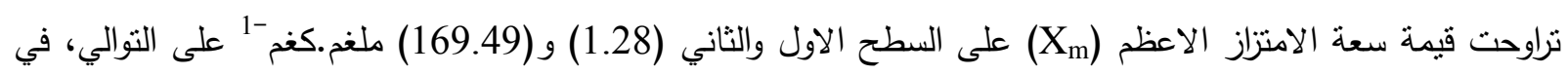
حين بلغت طاقة الربط (K) على السطح الاول والثاني (186.62) و (29.50) لتر .ملغم -1 وعلى التوالي.
\end{abstract}

الكلمات الدالة: الامتزاز الحيوي، كادميوم، .FTIR Chara sp.

\section{المقدمة}

Division: Charophyta

يعود طحلب .Charasp

Class: Charophyceae

Order: Charales

Family: Characeae

Genus: Chara

حسب التصنيف (Zeneli and Kashta, 2016)و و(Pandey and Trivedi, 1983). 
يتكون الطحلب من محور قائم متفرع يتصل بالمادة الاساس بواسطة اشباه الجذور، الساق الرئيسي مقسم الى عقد وسلاميات وقد

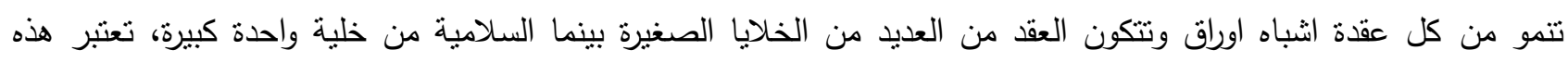
الطحالب .Chara sp حلقة الوصل بين الطحالب والحزازيات من الناحية النطورية. وهذه الطحالب تعيش في بيئة المياه العذبة وبعضها يفضل البيئة ذات الملوحة القليلة (عذبي، 2014). تعد المعالجة الحيوية (Bioremediation) هي عملية استخدام الكائنات الحية منل البكتيريا والخمائر والفطريات والطحالب والنباتات لعلاج الاوساط الملوثة، بما في ذلك الماء والتربة والهواء، من خلال تغيير الظروف البيئية لتحفيز نمو

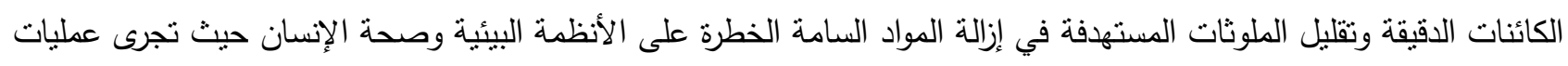
تحويل المركبات الملوثة من خلال التفاعلات التي تقوم بها الكائنات الحية كجزء من عمليات الأيض (محي الدين واخرون، الأنية . 2015

لوحظ في مواقع متعددة من العالم بما في ذلك أوربا وبدرجات متفاوتة من النجاح إن أحدى المشاكل الكبرى في المجتمعات التي تسودها الصناعة هي الاستخدام الكبير للمياه مع وجود منطلبات كثيرة للمياه النقية، لذلك استخدمت عدة أسناء أساليب لتقليل الاستهلاك المائي ولكن على المدى الطويل تبين أنه من المككن فقط أعادة تدوير مياه المجاري إلى مياه ذات نقاوة عالية (Kumar et al., 2011; Almukhtar and Ageena, 2012) تلائم المجتمعات الصناعية فضلاً عن الدول المتطورة لضمان نوفير مياه ذات جودة عالية وان التقنيات المتوافرة لمعالجة مياه المجاري لا تتواكب مع زيادة التلوث وخاصة المركبات العضوية التي زاد استخدامها في المجالات الصحية والمنزلية، وان التقنيات التي تستخدم فيها الاحياء المختلفة (كالجراثيم، الفطريات، الطحالب والنباتات) في معالجة وازالة الملوثات السامة (المواد العضوية، وادية غير العضوية والمعادن التقيلة) من المياه الملوثة، من افضل الطرائق الحديثة لمعالجة الملوثات، وتتميز بكونها امنة للبيئة وقليلة التكلفة مقارنة بالتقنيات الاخرى (طلعت، 2012؛ Kelly-Vargas et al., 2012 ؛ الحسيني والميالي، 2015).

اجريت العديد من الدراسات حول وجود العناصر الثقيلة في المياه المحلية كالكادميوم ، النحاس ، الرصاص، الخارصين، الزئبق، الكروم، القصدير والنيكل، إذ قامت الطائي (2012) بتقييم نبات عدس الماء في ازالته للعناصر الثقيلة (الزنك، الرصاص ،

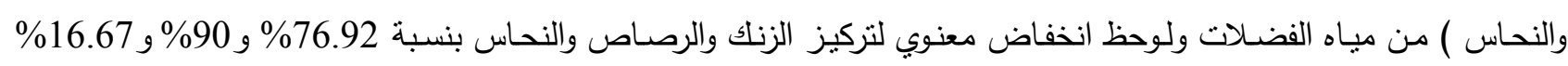

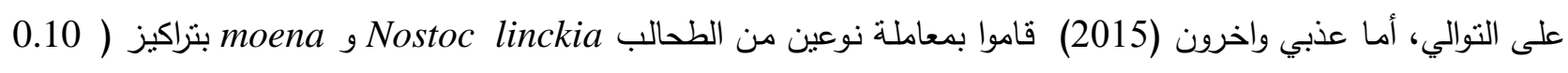

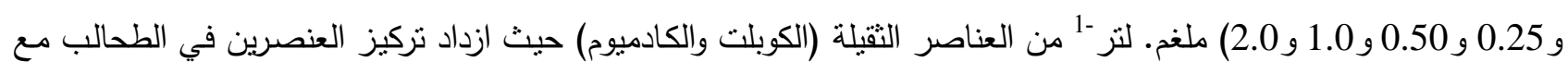

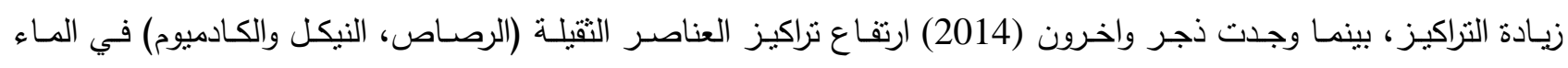
وطحلب .Chara sp في مياه الفضلات الناتجة من معامل انتاج البطاريات. اتجهت البحوث والدراسات حول إمكانية إستعمال أنواع الطحالب والسيانوبكتريا لإزالة المعادن الثقبلة من التزب الملوثية بسبب إمتلاكها آليات عديدة ومتتوعة في قابليتها على الامتصاص الحيوي والتراكم الحيوي داخل خلاياها إذ أظهرت كفاءة عالية

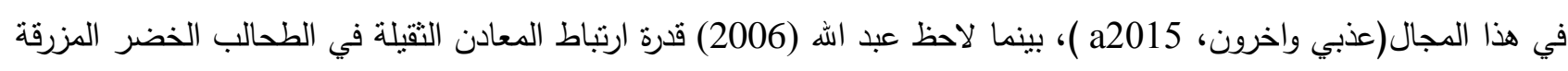

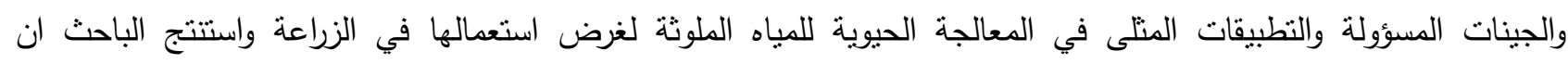

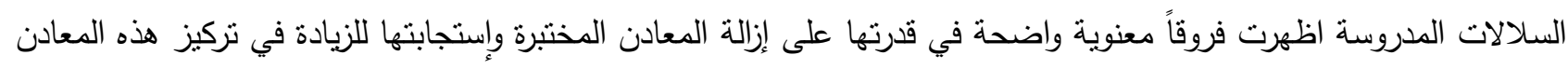
وإن Tolypthrix ceytonica يتبعها Anabaena varibilis اظهرت كفاءة عالية في التراكم الحيوي والقدرة على أزالة (100\%) للمعادن الثقيلة المختبرة. وأُختبرت أنواع من السيانوبكتريا في قابلية امنصاصها للمعادن الثقبلة من خلال استخدام معادن مفردة ونظام الإزالة لمعادن متعددة حيث اعطت نتائج إيجابية بسبب قدرة سلالات السيانوبكتريا المدروسة على إنتاج سكريات 
متعددة خارج خلوية (Pereira وآخرون،2011)، كما إستعملت مزارع السيانوبكتريا قي المعالجة الحيوية لمياه الفضلات الصناعية

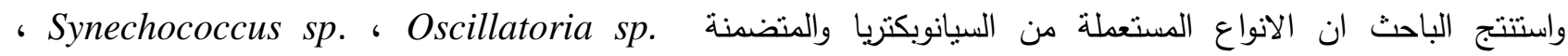
Cyanothece sp. Nostoc sp. ، Nodularia sp. بمعدلات سريعة وأعطت مدىً كبيراً في المعالجة الحيوية للتلوث الصناعي في مدينة بوبال الهندية (Dubey et al.,2011)، وفي وني دراسة تطبيقية في إستخدام الكتل الطحلبية كدلائل حيوية ومستخلصات نبانية للنلوث بالمعادن التقيلة في مياه الصرف الصحي لمدينة كامبالا في أوغندا حيث استخدمت نراكيز مختلفة من الرصاص، الكادميوم، النحاس والزنك مع كتل طحلبية وكانت النتائج انه عند استخدام الكتل الطحلبية اظهرت كفاءة عالية في تراكم المعادن الثقيلة وأن قيم التراكم الحيوي في الكتل الطحلبية للمعادن

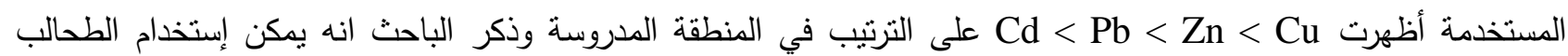
للمراقبة المائية ومرشحات حيوية نباتية للإستخلاص النباتي والكواشف الحيوية (Bioindicator) لتلوث مياه فضلات النظام البئي لمدينة كامبالا (Sekabira et al., 2011). وعليه كان الهدف من الدراسة الحالية هو استخدام طحلب .Chara sp كمعالج حيوي واختبار قدرته على إزالة تراكيز مختلفة من عنصر الكادميوم.

\section{المواد وطرائق العمل}

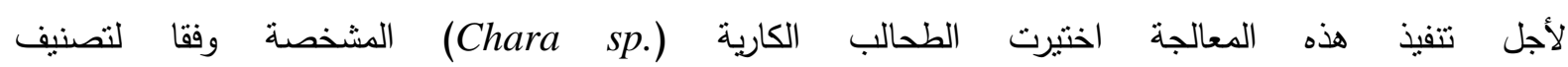
(Zeneli and Kashta,2016) و (Pandey and Trivedi, 1983) والموضحة بالثكل (1) وجفقت من خلال اخذ الكتلة الحية ووضعها على ورق نرشيح في غرفة حارة ومكان معقم لحين جفافها تماما واخذ منها وزن (2) غم ووضعت في أنابيب

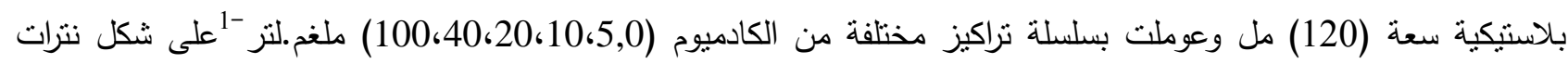
الكادميوم(CdNO التحضين والمنضمنة (24) ساعة، رجت لمدة نصف ساعة وتركت لمدة (24) ساعة للاتزان بعدها تم فصل رواشح الاتزان بواسطة الطرد المركزي لغرض تقدير الكادميوم فيها بجهاز مطياف الامتصاص الذري موديل (GBC 933plus) وبالطوال الموجي (nm326.10) مع وصفها رياضيا حسب معادلات الامتزاز الاتية : 


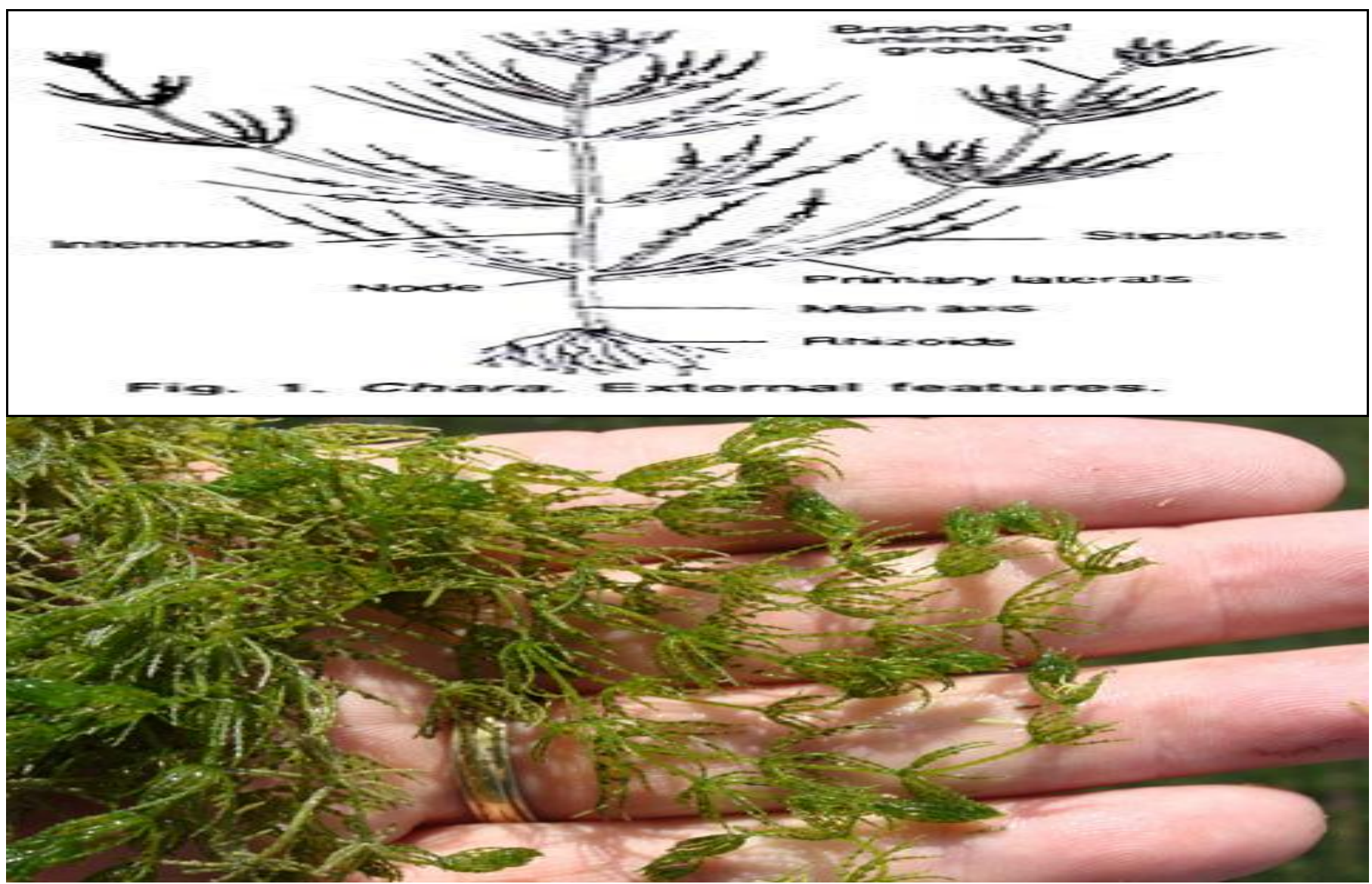

Chara sp. الثكل: 1 طحلب كارا

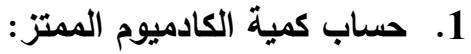

تم احتساب كمية الامتزاز للكادميوم عن طريق الفرق بين تركيز الكادميوم قبل وبعد الاتزان وحسب المعادلة التالية:

$$
\mathbf{C d}^{+2}-\mathbf{a d}=\frac{\mathbf{V}\left(\mathbf{C}_{\mathrm{in}}-\mathbf{C}_{\mathrm{fin}}\right)}{\mathbf{W}}
$$

اذ ان: و

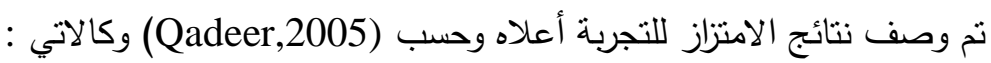

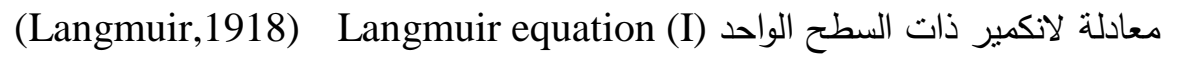

$$
\text { حيث ان: }
$$

=X K معادلة لانكماير ذات السطحين Langmuir,1918) Langmuir equation(II) 


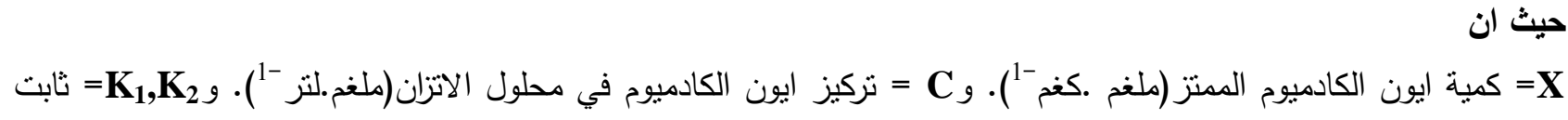

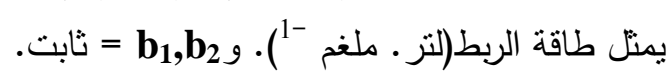

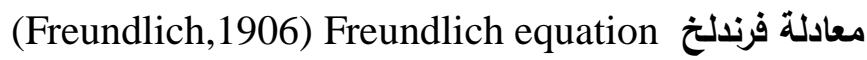

$\log X=b \log C+\log K$

حيث ان X (Temkin, 1934) Temkin equation معادلة تمكن

$\mathrm{X}=\alpha+\beta$ Lnc

حيث ان

= X ثوابت.

(Dubinin et al.,1947) Dubinin-Radushkevich equation معادلة دوبين

$\mathrm{Ce} / \mathrm{q}=1 / \mathrm{Kb}+1 / \mathrm{bCe}$

$\log q=\log K_{f}+n \operatorname{LogCe}$

$$
\begin{aligned}
& \text { حيث أن: } \\
& \text { تركيز النوع الايوني (الكادميوم) في محلول الاتزان. } \\
& \text { q } \\
& \text { = معامل التوزيع. و }=\mathbf{~}
\end{aligned}
$$

أجرى التحليل لمعامل الارتباط حسب برنامج Excel (2010). ولكون معادلتي الامتزاز ( لانكمير وفرندلخ) لا تعطيان

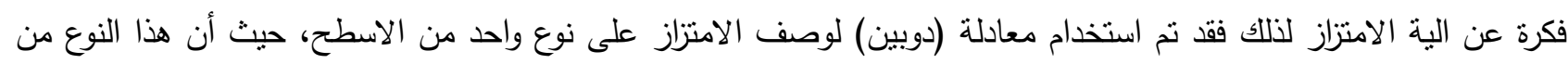

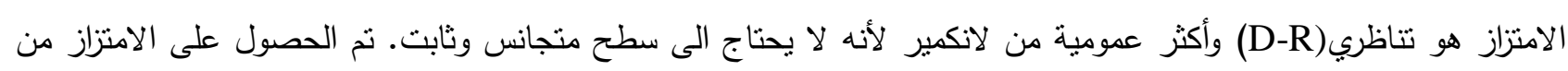

$\ln q=\operatorname{lnq}_{\mathrm{m}}-\mathrm{KE}^{2}$ المعادلة التالية :

$E=\left[R T \ln \left(1+\left(1 / C_{e}\right)\right]\right.$ 


\section{حيث أن :}

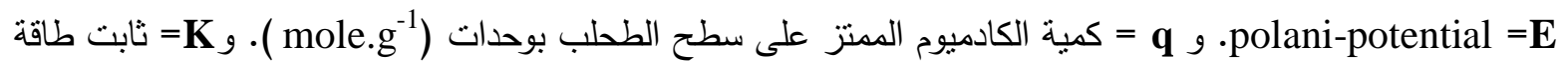

الامتزاز ويعبر عنه بوحدة)

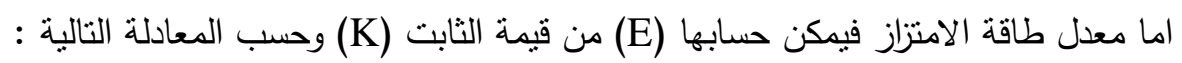

$$
\mathrm{E}=(-2 \mathrm{~K})^{-0.5}
$$

وتكمن أهمية حساب (E) هو للكثف عن نوع عملية الامتزاز فأن بلغت القيمة (8-16) كيلوجول مول-1 فأن عملية الامتزاز تفسر على أنها تبادل ايوني حسب (Kumar et al.,2010).

$\operatorname{lnq}=\operatorname{lnq}_{\mathrm{m}} \quad-\mathrm{KE}^{2}$

$\mathrm{E}=\left[\mathrm{RT} \ln \left(1+1 / \mathrm{C}_{\mathrm{e}}\right)\right]$

تم تحديد معايير الامتزاز (سعة الامتزاز وطاقة الربط) باعتماد الصيغة الخطية للمعادلات المذكورة اعلاه.

\section{فصص العينات بوساطة طيف الامتصلص للأثنعة تحت الحمراء Infrared Spectroscopy}

أُخذت عدة مليغرامات غير محددة الوزن من العينة المجفقة (طحلب كارا .Chara sp) ويتم فحصها بوساطة جهاز قياس

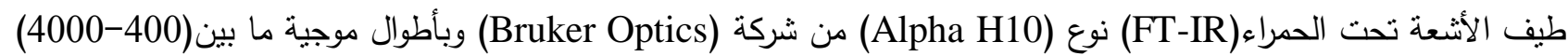

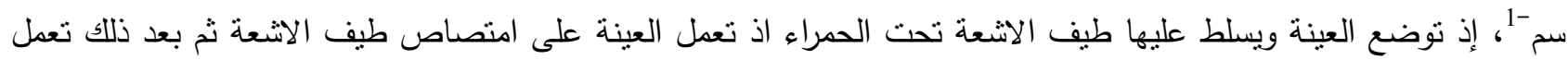
على تكون الحزم وتظهر على شكل مخططات وتقاس بـ (سم أ1).

\section{النتائج والمناقشة}

تشخيص المجاميع الفعالة:

تم تتخيص المجاميع الفعالة للطحلب .Chara sp المسؤولة عن الامتزاز باستخدام جهاز الاشعة تحت الحمراء والموضحة بالثكل(2) وهي : ( C=O ، COOH) والرابطة (C-H) الاليفاتية إضافة الى وجود مجاميع ذات شحنة سالبة مثل (OH) (C) )

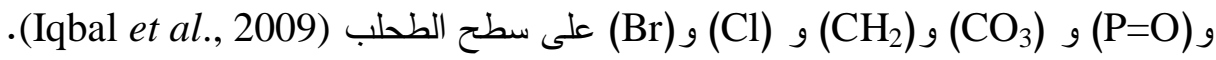




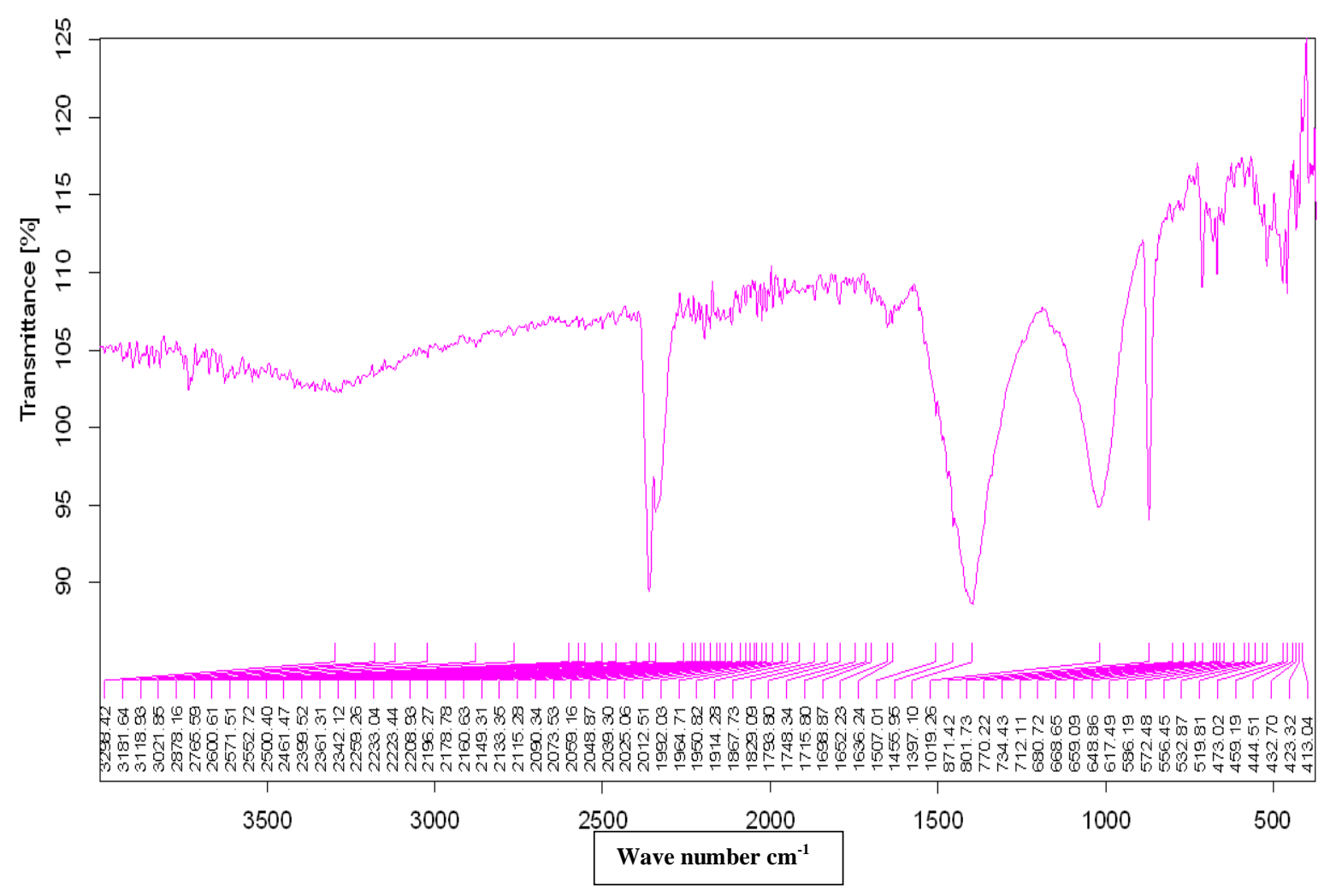

Chara sp. الشكل 2: طيف الاشعة تحت الحمراء لطحلب كارا

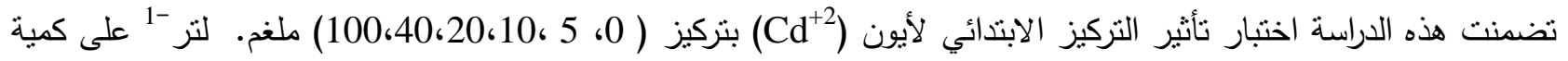

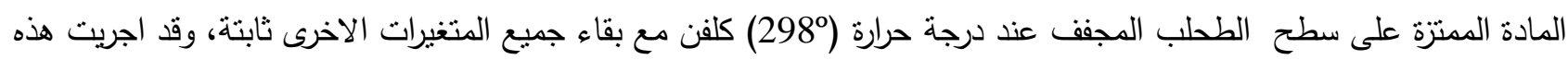
التجربة بطريقة الدفعة الواحدة وباستعمال المحلول الحاوي على الكادميوم، و (الجدول 1) يظهر مقارنة بين كمية المادة الممتزة من

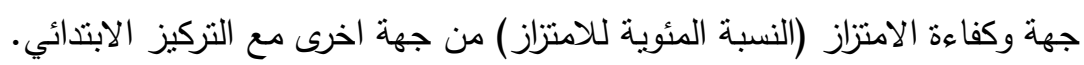

الجدول 1: يبين تغير الكمية الممتزة والنسبة المئوية للامتزاز مع التركيز الابتدائي عند (298) كلفن لمحلول ايون الكادميوم

\begin{tabular}{|c|c|c|}
\hline النسبة المئوية للامتزاز & الكمية الممتزة (ملفم. كفم -1) & التركيز الابتدائي المضاف(ملفم. لتر -1) \\
\hline- & -21.00 & (0 \\
\hline $\mathbf{7 8 . 8 0}$ & 78.80 & 5 \\
\hline 86.40 & 172.80 & 10 \\
\hline 88.75 & 355.00 & 20 \\
\hline 86.98 & 695.80 & 40 \\
\hline 85.62 & 1712.40 & 100 \\
\hline 85.31 & 602.96 & 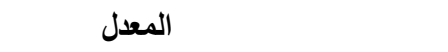 \\
\hline
\end{tabular}

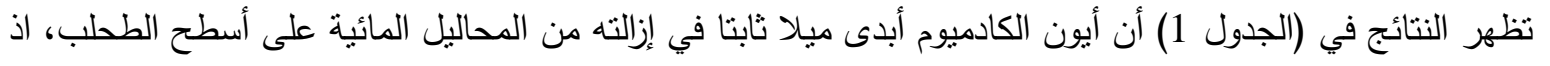
نلاحظ أن كمية المادة الممنزة والتي تعبر عن مقدار الإستصلاح البيولوجي (Bioremediation) تزداد مع زيادة التركيز

الابتدائي للفلز وكما هو مبين في الثكل (3). 


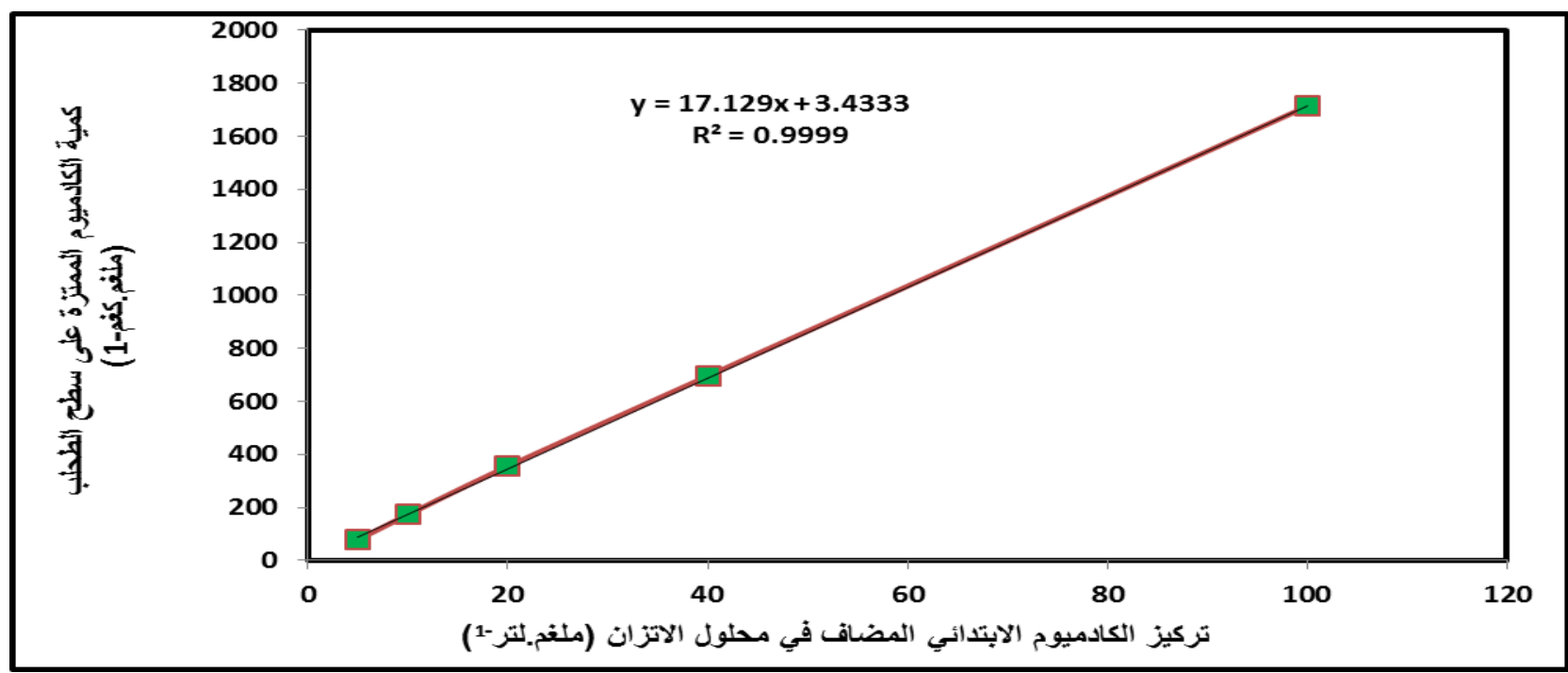

الشكل 3: يبين العلاقة بين تركيز الكادميوم الابتدائي في محلول الإتزان وكميته المتزة على سطح الطحلب عند درجة حرارة (298 $)$

وفي هذا الصدد كثنت العديد من الدراسات في مجال الامتصاص الحيوي للطحالب عن الألية المسؤولة عن هذا التفاعل والذي هو من نوع التفاعل التبادلي ما بين الايونات الموجبة للمعادن الخفيفة (كالسيوم، مغنيسيوم، صوديوم، بوتاسيوم) المرتبطة بالطحالب والعناصر الاخرى الموجودة في المحاليل المائية (Naja and Volesky, 2006) والذي يصف التطور في جزيئة الطحلب المازة من المحلول باتجاه موقع الأيون على الطحلب. ان هنالك عاملين رئيسين يحددان عملية الإمتزاز البيولوجي الأول يتمنل في قوة شحنة الأيونات (Electronegativity)

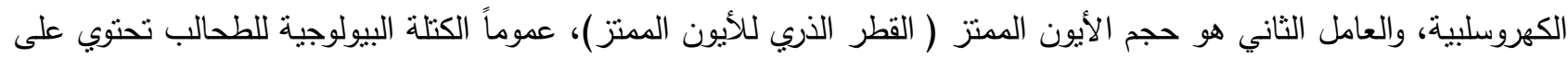
عناصر قاعدية (كالسيوم، مغنيسيوم ، صوديوم، بوتاسيوم) بالأصل موجودة في الماء الاعتيادي ولذلك عندما تتفاعل الكتلة

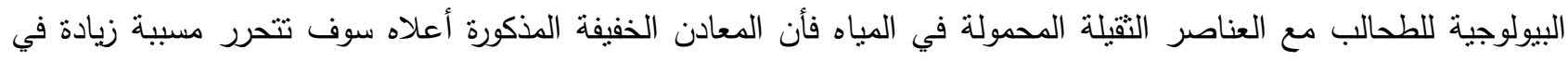
درجة تفاعل المياه مما يؤدي الى تكوين الأيونات القاعدية الخفيفة إضافة الى ذلك فإن تحرر هذه الايونات القاعدية سيزيد من

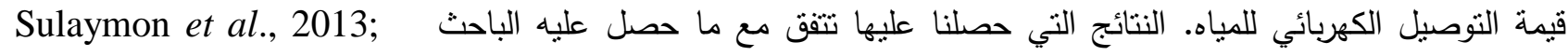
الذي وجد أن عملية الامتزاز البيولوجي للعناصر الثقبلة من قبل كتلة الطحالب أزاحت العناصر الخفيفة

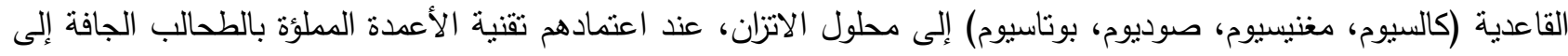
أن كمية العنصر الثقيل الممتز تعند على: 1. حالة الاتزان للامتزاز المتمانل حراريا. 2. سرعة الانتقال الكتلي. 3. العوامل الهيدروديناميكية (سمك عمود الإمتزاز ومدة التماس). إلا أن كفاءة الإزالة للطحلب تنتاقص مع زيادة التركيز الابتدائي وهذا يعزى إلى أن زيادة التركيز الابتدائي لعدد ثابت من

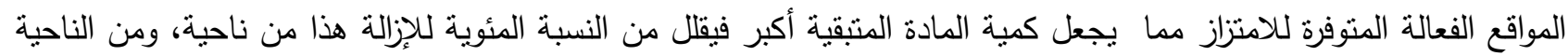
الثانية فان زيادة التركيز الابتدائي لأيون الفلز يزيد من تتافس هذه الأيونات للارتباط على العدد الثابت من المواقع الفعالة الفارغة

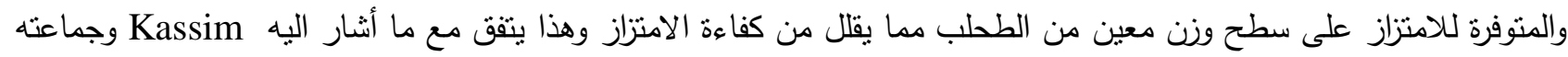
(2006) إلى قدرة الطحالب في إزالة (80\%) الى (100\%) من بعض العناصر النقيلة في أقل من يوم واحد، هذا الأمر يقلل من طاقة الارتباط بين أيون الفلز والموقع الفعال بسبب زيادة معامل فعالية السطح الماز . 
من المعروف أن عملية ازالة الفلزات النقيلة بواسطة الطحالب يمكن إن تعزى الى ميكانيكيات مختلفة حيث ترتبط هذه

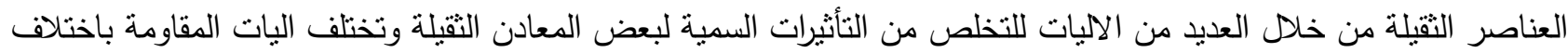
نوع الطحلب ونوع المعدن وقد أظهرت بعض أنواعها قدرتها على إفراز مركبات خارج خلوية(Extracellular compounds) لها ملان القدرة على الارتباط بالمعدن، وكما هو معلوم فإن للمعادن وأيوناتها قابلية الإتحاد مع المجاميع الوظيفية أو الروابط التي توجد

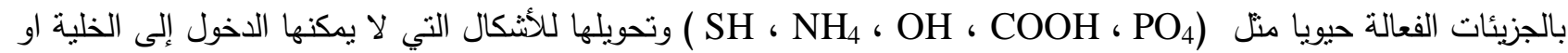

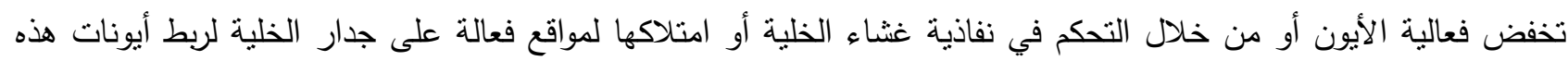
المعادن بجدار الخلية، إن هذه الأليات التي تستخدمها الطحالب في مقاومة التأثير السمي للمعادن أو منعها من الدخول إلى الخلية Shartooh et al., 2014; Farhan et ; Al-Qahtani, 2012 ; Kamsonlian et al., 2011) تعرف بالإقصاء أو الابعاد 2013.2al)، كما إن للعديد من أنواع الطحالب القدرة على إزالة التأثنر السمي داخل الخلية (Intracellular) بنكوينها مركبات بيتيدية غنية بـ (SH) تدعى (Y-Glutamyl-cystinil) في حالة التعرض للتراكيز الحادة من المعادن. أما عند مستوى التراكيز دون المميتة فيمكن أن نكون مركبات (YSH) (Y-Glutamyl- cystinil- cystinil) وفي كلتا الحالتين تعرف منل هذه بـان المركبات البيتيدية أو البروتينية بالمركبات المخلبية النباتية (Phytochelating compounds) وهي غنية (C) بانية بالحامض الأميني السستين (Cystine) ولها القدرة على ربط أيونات المعادن بتراكيبها الجزيئية عند مواقع (SH) الموجودة ضمن نركيبها الكيميائي

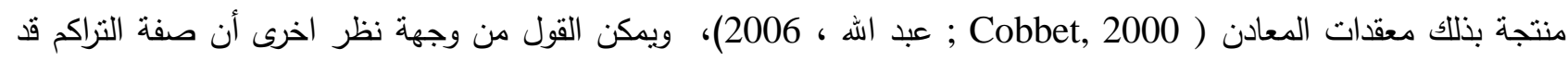
تعطي حلولاً وذلك بإستغلال كائنات مجهرية لها القدرة التكيفية على تحمل هذه النسب العالية من الملونات وتركيزها في أجسامها وبالتالي العمل على تخليص البيئة هنها وهذا ما يدعو الى التفكير بجدية بإستخدام هذه الكائنات كمعالجات أحيائية او كدلائل

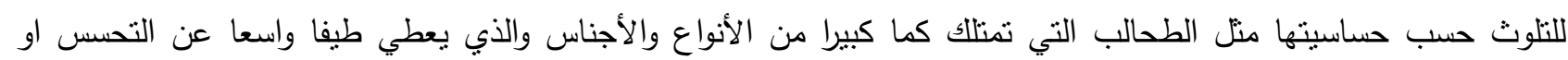

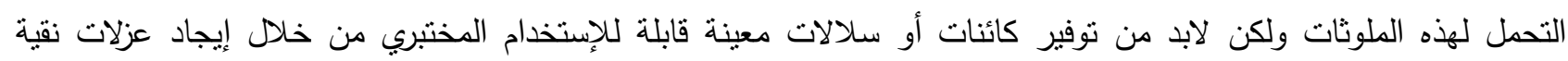
بإعتماد تقنية زراعة الطحالب، إذ تستخدم بعض أنواع الطحالب في صناعة منتجات لإزالة الزئبق من المياه حيث ينسبب الزئبق في حدوث طفرات وأضرار جينية (Sulaymon et al., 2013) مما يجعل للادنصاص الحيوي دورا هاما في حل مشكلة نلوث المياه من العناصر التقيلة والناتجة عن الفعاليات الصناعية حيث أن لها القدرة على ذلك (عذبي واخرون، 2015)، ومن هنا يمكن البحث عن اجناس تقوم بهذا العمل بشكل فعال. لذلك جاءت هذه الدراسة لغرض التعرف على إمكانية إزالة المعادن الثقبلة من

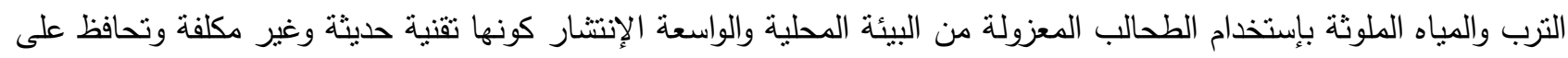
Wang and Chen, 2009; Al-Mayaly, 2009;) البيئة من التلوث وهذا ما أكدته العديد من الدراسات العالمية بهذا المجاه .(Ali,2011;Sulaymon et al., 2010 ; Rathinam et al., 2010 الوصف الرياضي لمعادلات الإمتزاز: تم اختبار قدرة الطحلب كارا على إمتزاز الكادميوم عن طريق الإتزان الديناميكي الدنماثل حراريا لأجل الحصول على ثوابت الإمتزاز البيولوجي (Biosorption) والتي تعبر عن خواص السطح الماز وميله أو درجة إنجذاب الأيونات التقيلة اليه كوسيلة معالجة للتخلص من العناصر النقبلة في المياه الثقبلة. يبين (الجدول 2) قيم معدل معامل التحديد (RE) والخطأ القياسي (SE) لمعادلات الإمتزاز والتي تم الحصول عليها بتطبيق معادلات (لانكمير ذات السطح الواحد وذات السطحين، فرندلخ، دوبين، تمكن وبولاني) على التوالي للكادميوم حيث يتضح من

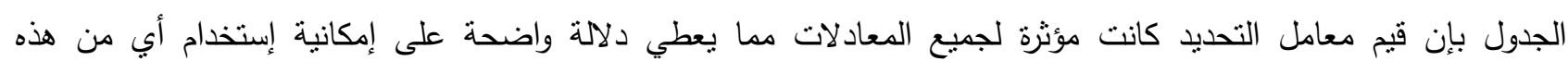

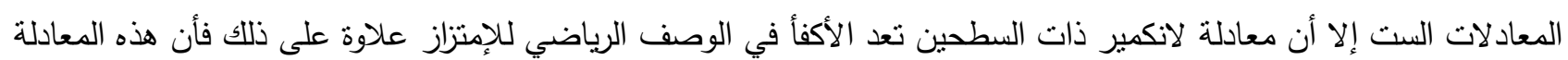


أظهرت تطابقاً كبيراً جدا لقيم الإمتزاز الفعلية والقيم المحسوبة من هذه المعادلة وذلك لإمتلاكها أقل خطأ قياسي مما يكسبها الأفضلية في الوصف الرياضي على بقية المعادلات.

\begin{tabular}{|c|c|c|c|}
\hline $\mathbf{R}^{2}$ & $\mathbf{S E}$ & & المعادة \\
\hline 0.87 & 0.002 & \multicolumn{2}{|c|}{ لانكمير ذات السطح الواحد } \\
\hline 1.00 & 0.000 & (السطح الاول) & \multirow{3}{*}{ لانكمير ذات السطحين } \\
\hline 1.00 & 0.0001 & (السطح الثاني) & \\
\hline 1.00 & 0.0001 & المعدل & \\
\hline 0.95 & 0.133 & \multicolumn{2}{|c|}{ فرندلخ } \\
\hline 0.95 & 0.133 & \multicolumn{2}{|c|}{ دوبين } \\
\hline 0.94 & 158.31 & \multicolumn{2}{|c|}{ تمكن } \\
\hline 0.93 & 0.36 & \multicolumn{2}{|c|}{ بولاني } \\
\hline
\end{tabular}

وإن تفوق معادلة لانكمير ذات السطحين في الوصف الرياضي لعملية إزالة (الإمتزاز البيولوجي) الأيونات من سطح

الطحلب نم توضيحه بالثكل (4) لمعادلة لانكمير ذات السطحين.

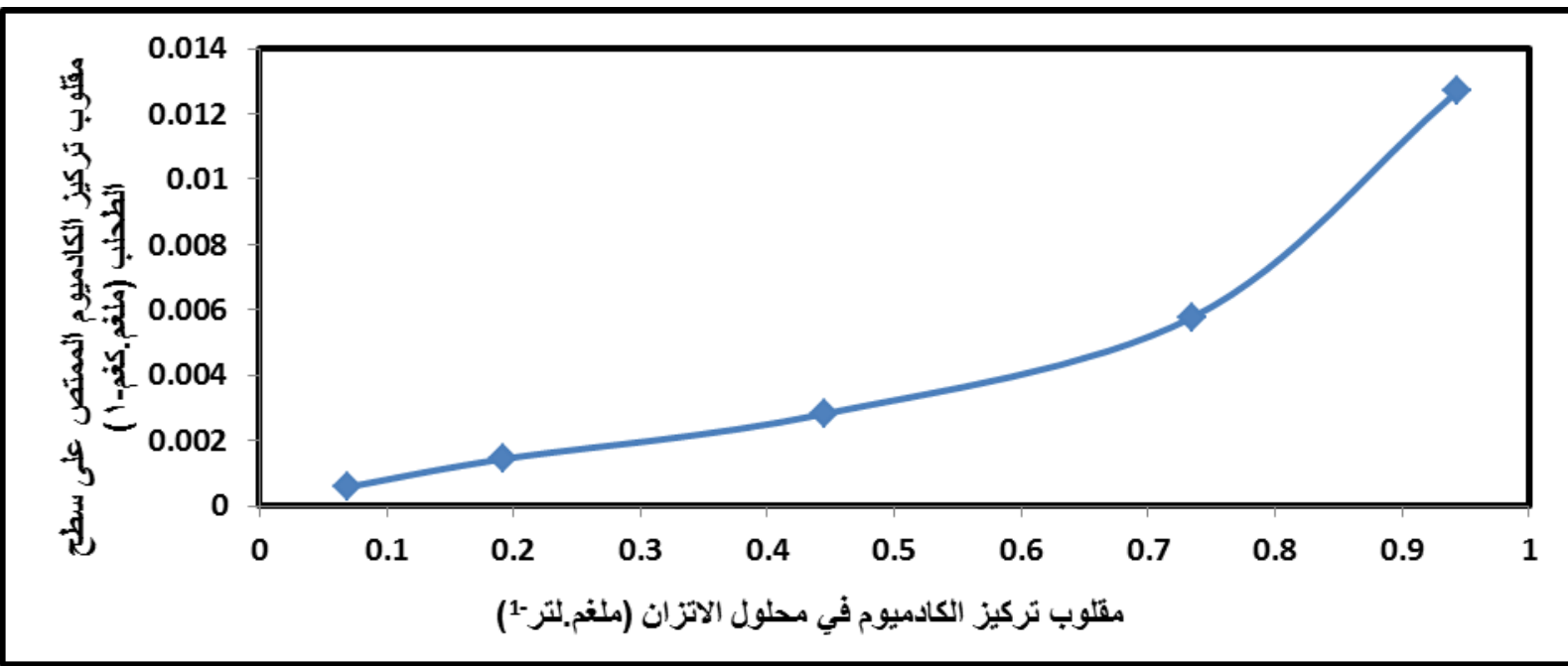

الثكل 4: العلاقة بين مقلوب تركيز الكادميوم في محلول الإتزان كدالة لمقلوب تركيز النوع الأيوني المدمص على سطح الطحلب

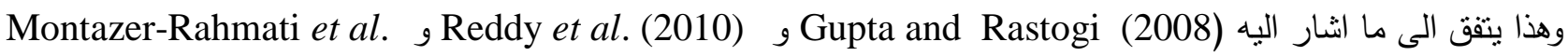

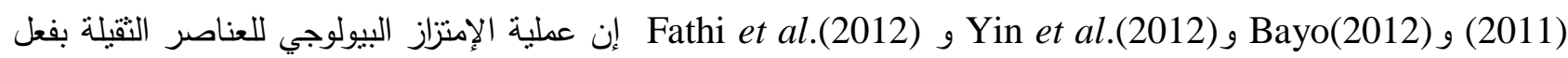
الأحياء الدقيقة تمر بمرحلتين الأولى إدمصاص سريع (Rapid uptake) يعزى إلى الإمتزاز السطحي على مكونات الجدران

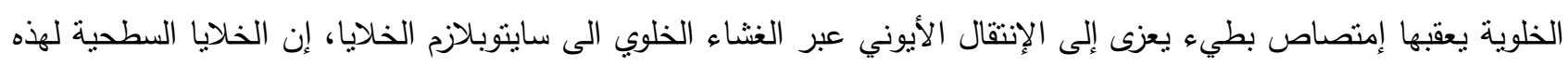


الاحياء تتألف من مركبات متعدد السكريات (polysaccharides) والبروتينات والليبيدات لذا تحوي على العديد من المجاميع

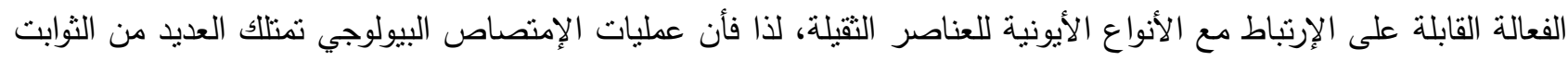
تعتمد على سرعة الإمتصاص البيولوجي (Biosorption) متل الخصائص التركيبية (Structural) لكل من المادة المازة والسطح العضوي الماز (Biosorpante) وعلى سبيل المثال وليس الحصر فإن كل من تركيب البروتينات وكثافة الثحنة السطحية والمساحة السطحية وطبيعة السطح (Topography) عوامل مؤثزة في عملية الامتزاز البيولوجي وكذلك فأن كمية المادة المازة ومدة التماس ودرجة تفاعل الوسط ودرجة الحرارة والتركيز الأولي للأيون المعدني لها تأثير كبير في سرعة علئ عملية (adsorpent)

الإمتزاز البيولوجي.

الامتزاز الأعظم للنوع الأيوني الثقيل على سطح الطحلب وطاقة ربطه:

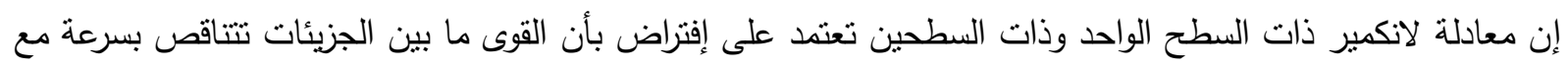

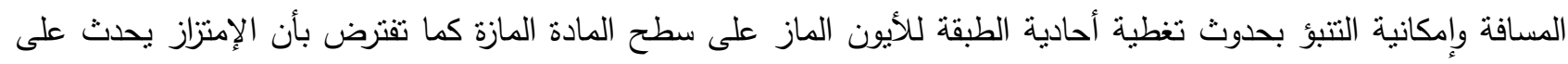
مواقع متخصصة ومتجانسة للمادة المازة إن قيم ثوابت الإمتزاز للصيغة الخطية لمعادلة لانكمير من قبل الطحالب تم إستخراجها

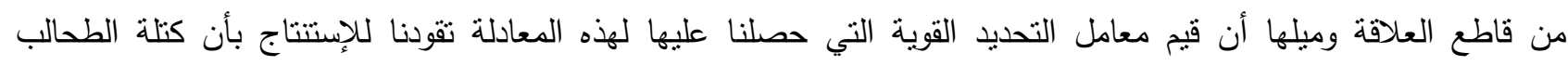
المستخدمة في الإمتزاز تخضع وبدرجة قوية لهذه المعادلة في عملية الإمتزاز ـ لقد تراوحت قيمة سعة الإمتزاز الأعظم على السطح الأول للطحالب الكارية (1.28) ملغم.كغمّ'، في حين بلغت طاقة الربط على السطح الأول للطحالب الكارية (186.62) لتز .ملغم -1 وكما هي موضحة في (الجدول 3).

الجدول 3 : يبين قيم ثوابت معادلة لانكمير ذات السطحين لإمتصاص الكادميوم على سطح الطحلب قيد الدراسة

\begin{tabular}{|c|c|c|c|}
\hline $\begin{array}{c}\mathbf{X}_{\mathbf{m} 2} \\
\left(\text { (ملغم.كغم }{ }^{1-}\right)\end{array}$ & $\begin{array}{c}\mathbf{K}_{2} \\
\left({ }^{-1-} \text { (لتز ملغم }\right)\end{array}$ & $\begin{array}{c}\mathbf{X}_{\mathbf{m} 1} \\
\left.\text { (ملغم.كغ }{ }^{-1-}\right)\end{array}$ & 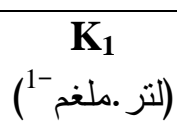 \\
\hline 169.49 & 29.50 & 1.28 & 186.62 \\
\hline
\end{tabular}

وبلغت قيم سعة الإمتزاز الأعظم على السطح الثاني للطحالب الكارية (169,49) (ملغم.كغم 1)، في حين بلغت طاقة الربط على السطح الثاني للطحالب الكارية (29.50) لتر .ملغم -1 وكما هي موضحة في (الجدول 3). وهذا ما يؤكد بوجود سطحين

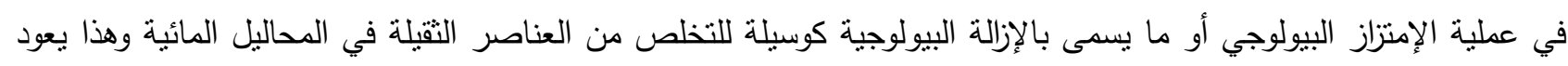

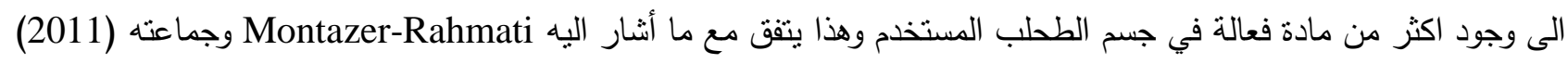

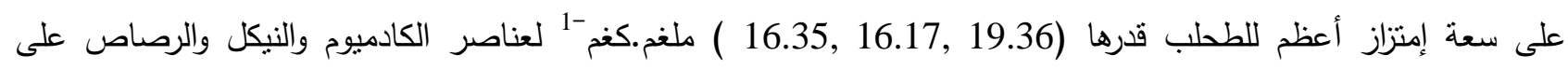
التوالي. وأثار هذا الباحث أيضا إلى نجاح معادلات لانكمير وفرندلخ وتمكن ودوبين في الوصف الرياضي لعملية الإمتزاز البيولوجي، .(Chakravarty et al.,2010; Babarinde,2011;Almasi et al.,2012; Tarbaoui et al.,2017) حيث حصل .Al-Khazragy et al (2005) إلى تفوق معادلة لانكمير على فرندلخ في الوصف الرياضي لعملية إمتزاز العناصر التقيلة من قبل الطحالب. 
أظهرت النتائج التي حصلنا عليها والموضحة في (الجدول 1) إختلافاً في سلوك وكميات الإمتزاز لكل أيون على سطح الطحلب وهذا الإختلاف يعود إلى الخواص الفيزيائية لكل أيون (السالبية الكهربائية ونصف القطر وجهد التأين) وبذلك يمكن

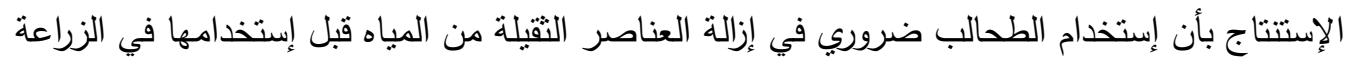

\section{المصادر العريية}

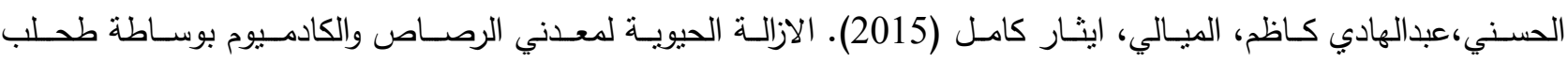
Westiellopsis prolifica

ذجر، لمياء عبد السـادة ؛الحسيني، احمد عيدان ؛ ناجي،هـاني سعيد ؛ طالب، رسل مكي (2014). الامتزاز الحيوي لعناصر الرصـاص والكـادميوم والنيكل مـن ميـاه الصـرف الصـناعي باسـتخدام الطحالب الكبيـرة الجافـة. مجلــة بغـداد للعلـوم. .1007-999،(2)11

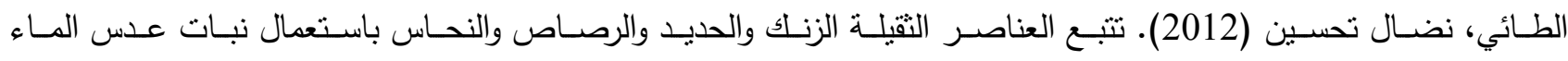
Lemna sp. الزراعية. 12 (2)،85-97.

طلعت، ريم أياد(2012). دراسة بيئية وبكتريولوجية لمياه الصرف الصحي لمصب قره سراي في مدينة الموصل وبعض تقنيات

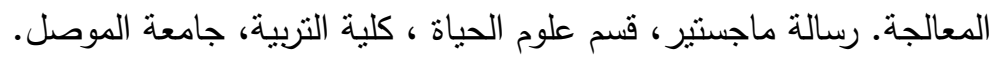

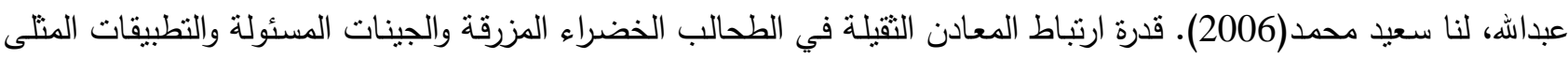
في المعالجة الحيوية للمياه الملوثة بغرض استخدامها بالزراعة. اطروحة دكتوراه، معهد البحوث والدراسات العربية، قسم

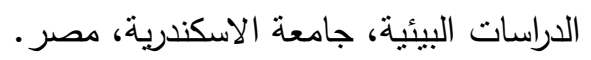

عذبي، احمد محسن؛ ناصـر، صباح هاني ؛ عيال، عبدالوهاب ريسـان (a2015). المعالجـة الحيويـة لبعض العناصر المعدنيـة

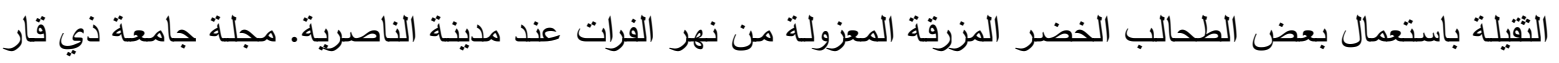

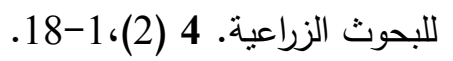

عذبي، احمد محسن؛ ناصر ، صباح ناهي ؛ عبال، عبدالوهاب ريسان (2015). دور بعض الطحالب الخضر المزرقة في المعالجة

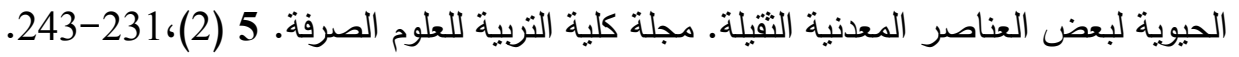

عذبي،احمد محسن(2014)."الطحالب العملي" . مطبعة دار الكتب للطباعة والنشر / جامعة البصرة.

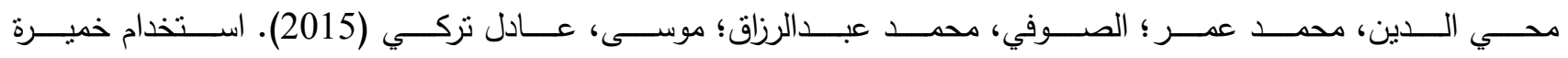

Saccharomyces cerevisiae

$.54-46 ،(2)$

\section{المصادر الأجنبية}

Ali, A.H. (2011). Performance of Adsorption/Biosorption for Removal of Organic and Inorganic Pollutants. Ph.D. Thesis, Univ. of Baghdad, College of Engineering.

Al-Khazragy, S.M.; Hussein, H.K.; Shareef, N.K. (2005). Efficacy of Spirogyrra spp. in adsorption of Cadmium, Cobalt, Copper, Iron, Lead, Mercury, Nickel, and Zinc Ions from aqueous solution. J. the Univ. of Kerbala, 3(11), 1-15.

Almasi, A.; Omidi, M.; Khodadadian, M.; Khamutian, R.; Gholivand, M.B. (2012). Lead(II) and Cadmium(II) removal from aqueous solution using processed walnut shell: kinetic and equilibrium study. Toxicol. Environ. Chem. 94,660-671. 
Al-Mayaly, I. K. (2009). Removal of Nickel ions by Scenedesmus quadricauda from contaminated water under laboratory conditions . Iraqi J. Sci., 50(4), 458-461.

Almukhtar, R.; Ageena, S.; Nagham, A. (2012). Water recycling / reuse in factories case study soft drink factory . Eng. and Tech. J. 30(1),1-14.

Al-Qahtani, K.M. (2012). Biosorption of binary mixtures of heavy metals by medicago sativa. World Appl. Sci. J., 16 (3), 465-473.

Babarinde, N.A.A. (2011). Kinetic, equilibrium and thermodynamic of the biosorption of $\mathrm{Pb}(\mathrm{II})$, $\mathrm{Cd}(\mathrm{II})$ and $\mathrm{Cr}(\mathrm{III})$ by neem leaf. J. Innovative Res. Eng. Sci., 2,291-306.

Bayo, J. (2012). Kinetic studies for Cd(II) biosorption from treated urban effluents by native grapefruit biomass (Citrus paradisi L.): The competitive effect of $\mathrm{Pb}(\mathrm{II}), \mathrm{Cu}(\mathrm{II})$ and $\mathrm{Ni}(\mathrm{II})$. Chem., Engineering J., 191, 278-287.

Bulgariu, D.; Bulgariu, L. (2012). Equilibrium and kinetics studies of heavy metal ions biosorption on green algae waste biomass. Bioresour. Technol.,103,489-493.

Chakravarty, P.; Sarma, N.S.; Sarma, H. (2010). Biosorption of cadmium(II) from aqueous solution using heartwood powder of Areca catechu. Chem. Eng. J., 162,949-955.

Cobbett, C.S. (2000). Phytochelatins and their role in Heavy Metal Detoxification. Plant Physiology, 123, 825-832.

Dubey, S. k.; Dubey, J.; Mehra S.; Tiwari, P.; Bishwas A. J. (2011). Potential use of cyanobacterial species in bioremediation of industrial effluents. African J. Biotechnology, 10(7), 11251132.

Dubinin, M.M.; Zaverina, E.D.; Radushkevich, L.V. (1947). Sorption and structure of active carbons. I. Adsorption of organic vapors Zh. Fiz. Khim., 21 , 1351-1362.

Farhan, A.M.; Al-Dujaili, A.H.; Awwad, A.M. (2013). Equilibrium and kinetic studies of cadmium(II) and lead(II) ions biosorption onto Ficus carcia leaves. Intern. J. Industrial Chem., 4(24), 1-8.

Fathi, A.A.; Azooz, M.M.; Al-Fredan, M.A. (2012). Abolishing toxicity of copper by some environmental factors using green alga Chlorella vulgaris. Am. J. Environ. Sci., 8, 633-641.

Freundlich, H.M.F. (1906). Over the adsorption in solution. J. Phys. Chem., 57, 385-471 .

Gupta, V. K.; Rastogi, A. (2008). Biosorption of Lead(II) from aqueous solutions by non-living algal biomass Oedogonium sp. and Nostoc sp.-A comparative study. Colloids and Surfaces B: Biointerfaces. 64,170-178.

Iqbal, M.; Saeed, A.; Zafar, S.I. (2009). FTIR spectrophotometry, kinetic and adsorption isotherms modeling, ion exchange and EDX analysis for understanding the mechanism of $\mathrm{Cd}^{2+}$ and $\mathrm{Pb}^{2+}$ removal by mango peel waste. J. Hazard Mater 164,161-171.

Kamsonlian, S.; Suresh, S.; Majumder, C.; Chand, S. (2011). Characterization of Banana and Orange Peels: Biosorption Mechanism. Intern. J. Sci. Technol. and Manag. 2 (4), 1-7.

Kassim, T.I.; Al-Rikabee, S.A.W.; Al-Rubaiee,G.H. (2006). Ability of Cyanophyceae species (Oscillatoria pseudogeminata and Spirulina major) in reduction of some pollutants from wastewater treatment plant, South Baghdad. Euro-Arab Environment Conference and Exhibition 2006, 612-621.

Kelly-Vargas, K.; Cerro-Lopez, M.; Reyna-Tellez, S.; Bandala, E.R.; Sanchez Salas, J.L. (2012). Biosorption of heavy metals in polluted water using different waste fruit cortex. Phys. Chem. Earth. 39,26-39.

Kumar, A.; Bisht, B.S.; Joshi, V.D.; Dhewa, T. (2011). Review on Bioremediation of Polluted Environment: A management Tool. Intern. J. Environ. Sci., 1(6).

Kumar, P.S.; Ramalingam, S.; Senthamarai, C.; Niranjanaa, M.; Vijayalakshmi, P.; Sivanesan, S. (2010).Adsorption of dye from aqueous solution by cashew nut shell: Studies on equilibrium isotherm, kinetics and thermodynamics of interactions. Desalination, 261,5260 . 


$$
\text { عبدالستار جبير زبن الحياني واخرون }
$$

Langmuir, I. (1918). The adsorption of gasses on plane surfaces of glass, mica and platinum. $J$. Am. Chem. Soc. 40, 1361-1368.

Montazer-Rahmati, M.M.; Rabbani, P.; Abdolali, A.; Keshtkar, A.R. (2011). Kinetics and equilibrium studies on biosorption of cadmium lead, and nickel ions from aqueous solutions by intact and chemically modified brown algae. J. Hazard. Mater. 185,401-407.

Naja, G.; Volesky, B. (2006). Behavior of mass transfer zone in a biosorption column. Environ. Sci. Technol. 40(12), 3996-4003.

Pandey, S.N.; Trivedi, P.S. (1983). Tikret University Textbook of botany. Printed by typographers at Rashtravani printers (India) $509 \mathrm{p}$.

Pereira, S.; Micheletti, E.; Zille, A.; Santos, A.; Moradas-Ferreira, P.; Tamagnin, P.; De Philippis, R. (2011). Using extracellular polymeric substances (EPS)-producing cyanobacteria for the bioremediation of heavy metals: do cations compete for the EPS functional groups and also accumulate inside the cell. Microbiology. 157, 451-458.

Qadeer, R. (2005). Adsorption of ruthenium ions in activated charcoal: influence of temperature on the kinetics of the adsorption process. J. Zhejiang University Science. 5,353-356.

Rathinam, A.; Maharshi, B.; Janardhanan, S.K.; Jonnalagadda, R.R.; Nair, B.U. (2010). Biosorption of cadmium metal ion from simulated wastewaters using Hypnea valentiae biomass: A kinetic and thermodynamic study. Biores. Technol., 101, 1466-1470.

Reddy, D.H.K.; Harinath, Y.; Seshaiah, K.; Reddy, A.V.R. (2010). Biosorption of Pb(II) from aqueous solutions using chemically modified Moringa oleifera tree leaves. Chem. Eng. J. 162,626-634.

Sekabira, K., Oryem,O. H.; Basamba, T.A.; Mutumba, G.; Kakudidi, E. (2011). Application of algae in biomonitoring and phytoextraction of heavy metals contamination in urban stream water. Int. J. Environ. Sci. Tech. 8(1), 115-128.

Shartooh, S. M.; Kasim, S.A.; Obaid, R.H.; Hadi, A.A.; Abdulmajeed, A.A. (2014). Lettuce leaves as biosorbent material to remove heavy metal ions from industerial wastewater. J. Baghdad for Sci. 11(3), 1164-1170.

Sulaymon, A.H.; Mohammed, A.A.; Al-Musawi, T.J. (2013). Multicomponent Biosorption of Heavy Metals Using Fluidized Bed of Algal Biomass. J. Engineering, 4(19), 469- 484.

Sulaymon, A.H.; Ebrahim, S.E.; Abdullah, S.M.; Al-Musawi, T. (2010). Removal of Lead, Cadmium, and Mercury Ions Using Biosorption. Desalination and Water Treatment 24,344-352.

Tarbaoui, M.; Oumam,M.; Benzina, M.; Bennamara, A.; Abourriche, A. (2017). Development of a new biosorbent based on the extract residue of marine alga Sargassum vulgare: application in biosorption of heavy metals. New Springer J.: Euro-Mediterranean J. for Environmental Integration View Project. I (4),1-13

Temkin, M. (1934). Die gas adsorption under nernstschewärmesatz. Acta. Physicochem. URSS, 1, 36-52.

Wang, D.; McLaughlin, E.; Pfeffer, R.; Lin, Y.S. (2011). Aqueous phase adsorption of toluene in a packed and fluidized bed of hydropholic aerogels. Chemical Engineering. 168, 1201-1208.

Wang, J.; Chen, C. (2009). Biosorbents for heavy metals removal and their future. Biotechnol. Advances, 27, 195-226.

Yin, P.; Wang, Z.; Qu, R.; Liu, X.; Zhang, J.; Xu, Q. (2012). Biosorption of heavy metal ions onto agricultural residues buckwheat hulls functionalized with 1Hydroxylethylidenediphosphonic Acid . J. Agric. Food Chem., 60,11664-11674.

Zeneli, V.; Kashta, L. (2016). Some Charophyta (Charales) from Coastal Temporary Ponds in Velipoja Area (North Albania). J. Environ. Sci., and Engineering B., 5., 69-77. 


\title{
Identitying The Absorbtion Range of Chara sp. Algae for Different Concentrations of Cadmium
}

\author{
Abdul Sattar J. Z. Al- Hayani \\ Department of Environmental Technology/College of Environmental Sciences and Technology / \\ University of Mosul \\ Abdelmoneim M. A. Kannah \\ Department of Biology / College of Science / University of Mosul \\ Hiba K. Saeed \\ Department of Biophysics Science / College of Science / University of Mosul
}

\begin{abstract}
The adsorption of the cadmium element ion was used by the Chara sp., where it was dried and the effective groups of algae responsible for adsorption using infrared (FTIR) were $\mathrm{COOH}$ ، $(\mathrm{C}=\mathrm{O})$ and aliphatic nexus $(\mathrm{C}-\mathrm{H})$, in addition to the presence of groups with a negative charge such as $(\mathrm{OH}),(\mathrm{P}=\mathrm{O}),\left(\mathrm{CO}_{3}\right),\left(\mathrm{CH}_{2}\right),(\mathrm{Cl})$ and $(\mathrm{Br})$ on the surface of the algae, and taking them at a weight of $2 \mathrm{~g}$ and treated with a series of different concentrations $(0,5,10,20,40,100) \mathrm{mg} . \mathrm{L}^{-1}$ of Cadmium ion where incubation coefficients at $\left(298^{\circ}\right)$ Kelvin,

After the incubation period, the stabilizers were extracted and Cadmium was quantified with mathematical description according to the equations of the single-surface (Langmuir, Freundlich, Temkin, Dubinin and Polani) respectively for Cadmium, It was found that the values of the coefficient of determination were effective for all equations, which gives a clear indication of the possibility of using any of these six equations. However, the two-sided Langmuir equation is the most efficient in the mathematical description of adsorption. Moreover, this equation showed a very large correlation between the actual adsorption values and the calculated values of this equation because they have the lowest standard error (SE) (0.0001) and the highest coefficient of determination $\left(\mathrm{R}^{2}\right)$ (1.00), which gains the advantage in the mathematical description on the rest of the equations. The maximum adsorption capacity $(\mathrm{Xm})$ on the first and second surfaces (1.28) and (169.49) $\mathrm{mg} \mathrm{Kg}^{-1}$ respectively, while the binding capacity $(\mathrm{K})$ on the first and second surfaces (186.62) and (29.50) L. $\mathrm{mg}^{-1}$ respectively.
\end{abstract}

Keywords: Bioadsorption, Cadmium, Chara sp. , FTIR 